\title{
Quick Review: Melanoma
}

\section{Perry, B Phillips}

\section{Citation}

C Perry, B Phillips. Quick Review: Melanoma. The Internet Journal of Dermatology. 2001 Volume 1 Number 2.

DOI: $\underline{10.5580 / 178 \mathrm{a}}$

\begin{abstract}
Melanoma is

diagnosed in 30,000 Americans per year. The incidence of melanoma has been increasing over the past ten years at an alarming rate (83\%). While melanoma accounts for only $3 \%$ of all cutaneous neoplasms, it is linked to $65 \%$ of skin cancer deaths. Melanoma is thought to be linked to ultraviolet radiation exposure, especially UV-B radiation [280-320 nm], and fair-skin. Patients that burn easily are at greater risk than those who tan easily. Non-Caucasians have a lower incidence of melanoma as well. Melanoma can be effectively treated with excision. Adjuvant therapy for node positive patients and those with deeper lesions is available. Prevention and early detection remain the best therapies.
\end{abstract}

\section{DIAGNOSIS}

\section{A B C D}

Any skin lesion with Asymmetry, Border irregularity, Color variation, Diameter greater than $6 \mathrm{~mm}$ should raise suspicion of a neoplastic process.

\section{RISK FACTORS}

Fair skin individuals who burn easily are more likely to develop melanomas. $10 \%$ of melanomas occur in patients with a strong familial history.

Patients with multiple nevi and dysplastic nevi (5-20\% more risk than the general population) are also more at risk for melanoma.

\section{APPROACH}

A complete medical history is obtained with special attention to familial history of skin malignancies, sun exposure as a child (number of sunburns as an adolescent) followed by a complete physical examination including a thorough skin and lymphatic drainage basin assessment.

Incisional or excisional biopsy must then be obtained (make sure to include all of the dermis and into the subcutaneous tissue).

\section{STAGING: CLARK LEVELS}

1. Contained within the epidermis

2. Penetration into papillary dermis

3. Through papillary dermis to reticular dermis

4. In the reticular dermis

5. Into subcutaneous tissue

Clark classified lesions based on depth because of the associated prognosis.

Clarks level I lesions are not considered malignant and are completely treated by excision. Once the lesion penetrates into the papillary dermis it has potential to metastases.

Survival by stage II, III, IV, V is $88 \%, 66 \%, 54 \%$, and $22 \%$ at 5 year follow up.

The difficulty with this staging system is the variability in pathological examinations specifically concerning histology of the papillary and reticular dermis.

Breslow (like Clark) knew that the depth of penetration into normal tissue was inversely related to survival. Additional investigations have indeed correlated depth of invasion in a linear relationship to morbidity over a ten year period. 
Breslow devised a staging system that used a micrometer and classified the tumor based on invasion depth. Tumors less than $0.75 \mathrm{~mm}$ in depth were curable with simple excision. Those over $0.75 \mathrm{~mm}$ in depth had potential for metastases with worsening prognosis.

American Joint Committee on Cancer
IA: T1 No Mo
IB: T2 No Mo

IIA: T3 No Mo

IIB: T4 No Mo

\section{III: Tx N1 Mo}

\section{IV: Tx N2 Mo or Tx Nx M1}

Sites of Metastases:

Likelihood of lymph node and distant metastases based on depth:

\section{Some Points}

1. Most common cause of skin cancer related deaths.

2. Early detection and excision offers best chance for cure.

3. Depth of invasion linearly related to mortality.

4. Depth of invasion $>1.5 \mathrm{~mm}$ has a $50 \%$ chance of having lymph node metastasis.

5. Most common site of metastasis; skin, lymph nodes, lung, liver, brain, bone, and intestine.

\section{THERAPY - IN GENERAL}

Survival Facts:

Poor Prognostic Factors
- Lesions with ulceration.

- Male gender.

- Lesions on the trunk, scalp, hands, or feet.

\section{LOCAL THERAPY}

Once the initial biopsy reveals melanoma and its depth is assessed, a therapeutic plan can be formulated. If adenopathy is detected in the lymphatic drainage basin one proceeds with lymph node dissection and extirpation.

If the lesion penetrates more than $0.75-1 \mathrm{~mm}$ and the lymphatic drainage basin is clinically negative a sentinel lymph node sampling can be preformed to assess the nodal status.

If the sentinel node returns with metastatic melanoma, the remaining lymphatic drainage in that basin is subsequently extirpated. There is still some debate as to the utility of complete lymph node dissection and its affect on long term survival. Once the lymphatic are addressed, the lesion is locally re-excised with appropriate margins based upon location and depth of penetration.

It may be necessary to consult with a plastic surgeon for difficult closures.

\section{ADJUVANT THERAPY}

For patients with stage III disease immune modulation with interferon alpha for a period of one year is offered.

Other regiments include DTIC, BCG injections, IL-2 injections and numerous clinical trials. Response rates remain dismal at $10-20 \%$.

Radiation therapy may be helpful in those patients requiring palliation.

\section{References}




\section{Author Information}

Charles W Perry, MD

Dept. of Surgery, University of Arizona

\section{Bradley J. Phillips, MD}

Dept. of Trauma \& Critical Care, Boston University School of Medicine, Boston Medical Center 